\title{
Ventricular perforation as a complication of percutaneous valve replacement
}

\author{
Cornelis de Jager, BSC \\ College of Medicine, University of Saskatchewan, Saskatoon, Canada

\section{Ivan L Norval, MB ChB} \\ Jennifer R Tynan, MD, FRCP (C) \\ Department of Medical Imaging, Royal University Hospital, University of Saskatchewan
}

Corresponding author: C de Jager (cod204@mail.usask.ca)

\begin{abstract}
Aortic stenosis (AS) is the most commonly encountered valvular disease in developed countries. Once symptomatic, this disease carries a dismal prognosis. Patients unfit for surgical valve replacement may require transcatheter aortic valve implantations (TAVI). We present a complication of this novel approach where a prosthetic valve is delivered through the femoral artery by means of a Retroflex delivery system developed by Edwards Lifesciences.
\end{abstract}

\section{S Afr J Rad 2012;16(3):102-103. DOI:10.7196/SAJR.669}

An 81-year-old woman was admitted to hospital for symptomatic aortic stenosis (AS) in July 2010, with a history of moderate AS, mild-moderate aortic regurgitation, moderate mitral regurgitation, paroxysmal atrial fibrillation and pacemaker implantation for a 2:1 atrioventricular heart block. According to the New York Heart Association (NYHA) classification, she had class 4 symptoms despite a preserved ejection fraction of $65-70 \%$.

Owing to prior bilateral mastectomies, she was considered high risk for conventional surgery, with concern about wound complications of her sternum. It was felt that a transcatheter valve would be a safer option, and she underwent the transcatheter aortic valve implantation (TAVI) procedure in April 2011. She received an aortic $23 \mathrm{~mm}$ Edward Sapien XT (9300 TFX) prosthesis in what was described as a successful procedure at the time. Three days later, computed tomographic angiography (CTA) of the chest revealed a small left pleural effusion and a $5 \mathrm{~mm}$ rounded area of contrast in the epicardial space. Owing to the stable condition of the patient, the peri-procedural complication was deemed to be of uncertain significance.

Two days after discharge, she was re-admitted with signs and symptoms of hypovolaemia. Echocardiogram and computed tomography (CT) confirmed cardiac tamponade. An associated defect in the left ventricular free wall was visualised on CT, consistent with left ventricular perforation (Fig. 1). The patient developed bilateral pleural effusions over the interval, thought to be related to congestive heart failure (Fig. 3).

After treatment with diuretics and thoracocentesis, the patient was discharged, only to be re-admitted a week later for worsening bilateral pleural effusions. An echocardiogram showed self-sealing of

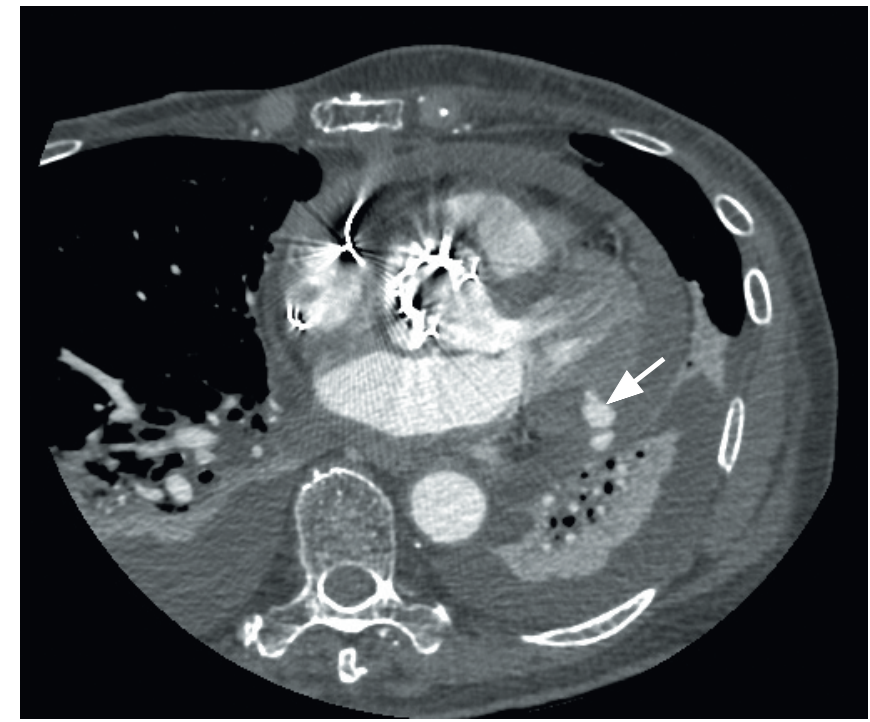

Fig. 1. Axial CT showing a thin tract of contrast extending into the myocardium and leading up to a small pseudoaneurysm in the posterolateral aspect of the left ventricular free wall.

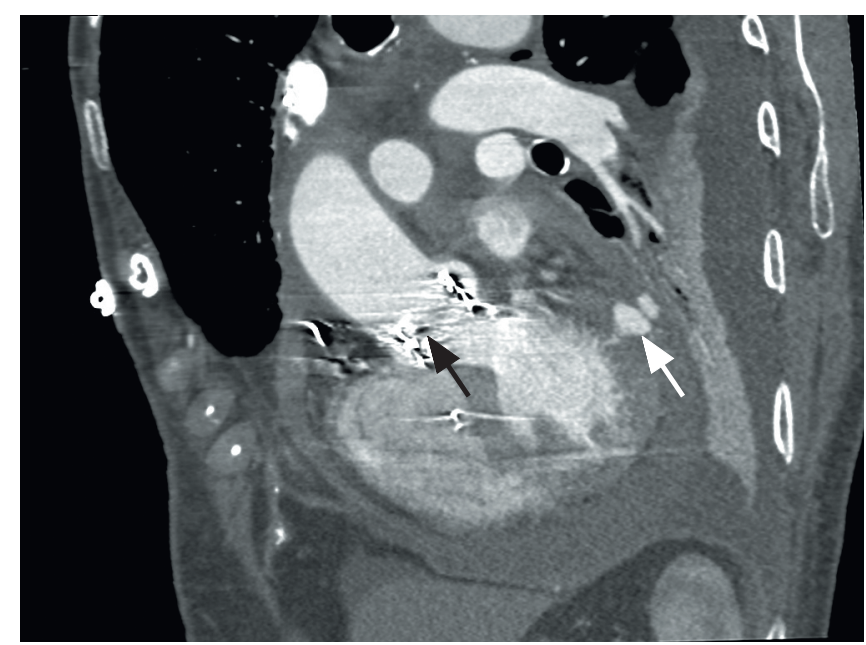

Fig. 2. Sagittal CT reconstruction of the thorax showing the implanted aortic valve. The left ventricle free wall defect with small pseudoaneurysm is also again noted. 


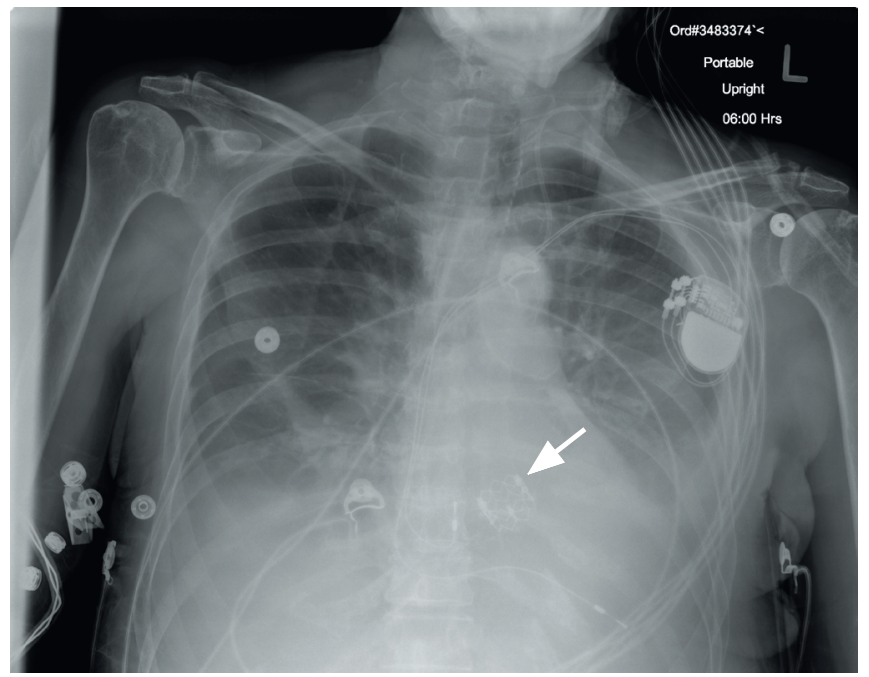

Fig. 3. AP radiograph showing valve prosthesis in situ.

the left ventricular free wall rupture and disappearance of tamponade physiology. At the 2-month follow-up, the patient was still being treated in hospital for pleural effusions by means of diuretics, thoracocentesis and supportive treatment.

\section{Discussion}

AS most often arises from age-related degenerative calcified changes to the valve, and is defined as the obstruction of blood flow through the aortic valve during left ventricular ejection. ${ }^{3} \mathrm{~A}$ transvalvular gradient is established when the valve orifice is reduced by more than $50 \%$ of its normal size. ${ }^{3}$ Significant elevation in left ventricular pressure is then needed to drive blood into the aorta. ${ }^{2}$ At first, the heart can compensate through concentric hypertrophy but, due to disease progression, becomes overwhelmed, causing a drop in the systolic left ventricular ejection fraction. ${ }^{3}$ Severe AS is graded as an orifice area $<1.0 \mathrm{~cm}^{2}(0.6$ $\mathrm{cm}^{2} / \mathrm{m}^{2}$ ), mean gradient $>40 \mathrm{mmHg}$ or peak jet velocity $>4 \mathrm{~m} / \mathrm{s}^{1,3}$

The current gold standard of treatment is surgical valve replacement. Many patients do not meet inclusion criteria or have overwhelming co-morbidities which make them unfit for surgery. ${ }^{5}$ This is unfortunate as 1-year mortality with medical management for inoperable AS exceeds $50 \%{ }^{6}$

Balloon valvulopasty has been used for symptomatic relief in inoperable patients until recently when TAVI was introduced as a new treatment option for patients who have inoperable AS. ${ }^{6}$ The first human valve implant was performed in 2002, and techniques and equipment have steadily improved since. ${ }^{4}$ While there are several new generations of valves in clinical trials, there are only 2 prosthetic valves currently in commercial use, known as the Metronic CoreValve and Edwards SAPIEN
XT.

According to the recently reported Placement of AoRTic TraNscathetER Valve Trial (PARTNER) study, TAVI dramatically reduces mortality at 1 year by an absolute $20 \%$ and improves quality of life. ${ }^{1,6}$ This means that only 5 patients have to undergo TAVI to save one life at 1 year. A multidisciplinary team usually performs the TAVI procedure in a cardiac catheter lab or hybrid operating room equipped with high-quality imaging. ${ }^{6}$ Conscious sedation with transthoracic echocardiography is an option although general anaesthesia may facilitate trans-oesophageal echocardiography and increase patient safety and comfort. Vascular access most commonly includes femoral and trans-apical (through anterolateral thoracotomy), although utilising subclavian access is becoming increasingly popular, especially with CoreValve implantation. ${ }^{6}$

Complications associated with TAVI most frequently include vascular events owing to large-calibre catheters, and used to range from $2-18 \% .5,6$ This number is falling due to improved techniques, screening, and the development and use of smaller catheters. ${ }^{6}$ Pericardial tamponade has been documented with the CoreValve to occur between 0 and $7 \%$, and is usually due to guidewire or manoeuvring thrusts to bring the prosthesis into the left ventricle that can potentially cause peforations. ${ }^{3}$ Clinical stroke reportedly complicates from $0-10 \%$ of TAVI procedures. ${ }^{5,6}$ Other complications include coronary occlusion, atrioventricular block (3 - 10\% of SAPIEN valves) and acute renal injury. ${ }^{5,6}$ Trace to mild aortic regurgitation post implantation is reported in up to $70 \%$ of cases. Moderate regurgitation is less common at $5-15 \%$, and severe regurgitation occurs in approximately $2 \%{ }^{6}$

\section{Conclusion}

TAVI is becoming an increasingly popular treatment for symptomatic aortic stenosis in patients unfit for conventional surgery. Although the procedure is less invasive than surgical valve replacement, significant risks are still involved with this form of treatment. It is of the utmost importance that radiologists are aware of possible complications and are able to identify them promptly, as early treatment could have a significant effect on patient outcomes.

1. Leon M, Smith C, Mack M, et al. Transcatheter aortic-valve implantation for aortic stenosis in patients who cannot undergo surgery. N Engl J Med 2010;363:1597-1607.

2. Lilly L. Pathophysiology of Heart Disease. 5th ed. Philadelphia: Lippincot Williams \& Wilkins, 2011:202204.

3. Spaccarotella C, Mongiardo A, Indolfi C. Pathophysiology of aortic stenosis and approach to treatment with percutaneous valve implantation. Circ J 2011;75:11-19.

4. Thomas, M. The global experience with percutaneous aortic valve replacement. JACC: Cardiovascular Interventions 2010;3(11):1103-1109.

5. Webb J, Cribier A. Percutaneous transarterial aortic valve implantation: what do we know? Eur Heart J 2011;32(2):140-147.

6. Willson A, Webb J. Transcatheter treatment approaches for aortic valve disease. Int J Cardiovasc Imaging 2011;27(8):1123-1132. 